\title{
Osteopontin splice variants are differential predictors of breast cancer treatment responses
}

Krzysztof Zduniak', Anil Agrawal ${ }^{2}$, Siddarth Agrawal ${ }^{2}$, Md Monir Hossain $^{3}$, Piotr Ziolkowski ${ }^{{ }^{*}}$ and Georg F. Weber ${ }^{4^{*}}$

\begin{abstract}
Background: Osteopontin is a marker for breast cancer progression, which in previous studies has also been associated with resistance to certain anti-cancer therapies. It is not known which splice variants may mediate treatment resistance.

Methods: Here we analyze the association of osteopontin variant expression before treatment, differentiated according to immunohistochemistry with antibodies to exon 4 and to the osteopontin-c splice junction respectively, with the ensuing therapy responses in 119 Polish breast cancer patients who presented between 1995 and 2008.

Results: We found from Cox hazard models, logrank test and Wilcoxon test that osteopontin exon 4 was associated with a favorable response to tamoxifen, but a poor response to chemotherapy with CMF (cyclophosphamide, methotrexate, fluorouracil). Osteopontin-c is prognostic, but falls short of being a significant predictor for sensitivity to treatment.
\end{abstract}

Conclusions: The addition of osteopontin splice variant immunohistochemistry to standard pathology work-ups has the potential to aid decision making in breast cancer treatment.

Keywords: Tumor progression marker, Immunohistochemistry, Breast cancer, Chemotherapy, Hormone therapy, Radiation therapy

\section{Background}

Biomarkers are important for guiding the diagnosis and treatment of cancer. Two broad groups comprise prognostic markers and predictive markers. Prognostic markers allow forecasts regarding the natural course of the disease. They differentiate between patients likely to have a good versus a poor outcome. By contrast, predictive markers provide upfront information regarding how likely a patient is to benefit from a specific treatment, and hence may guide the choice from available therapies. Anticipating treatment response or risk of treatment resistance is a critical need in cancer care. Relevant predictive markers mostly belong to the groups of drug targets, molecules associated with drug transport or metabolism, and

\footnotetext{
* Correspondence: ziolkows@interia.pl; georg.weber@uc.edu

${ }^{1}$ Department of Pathology, Wroclaw Medical University, Wroclaw, Poland

${ }^{4}$ University of Cincinnati Academic Health Center, College of Pharmacy, 3225

Eden Avenue, Cincinnati, $\mathrm{OH}$ 45267-0004, USA

Full list of author information is available at the end of the article
}

regulators of apoptosis or DNA repair. As such, they are mechanistically involved in the drug response. In addition, because highly aggressive tumors are generally more difficult to manage than less aggressive ones, some prognostic indicators may also have predictive properties.

In the histopathologic assessment of breast cancer, the standard markers ER, PR, and HER2 identify drug targets, as ER-positive tumors are candidates for antiestrogen treatment whereas HER2-positive tumors are candidates for treatment with trastuzumab. Further, the absence of all three marker molecules defines triplenegative breast cancers, which have a poor prognosis and limited treatment options. There is a lack of more refined predictive markers for treatment success in the disease.

In breast cancer, osteopontin is a biomarker for aggressiveness and for prognosis. Further, it has been described as a marker for treatment responses. Osteopontin causes 
breast cancer resistance to cyclophosphamide [1], doxorubicin [2-4], paclitaxel [4] and cisplatin [4] through its anti-apoptotic properties or through the upregulation of drug exporters. Its levels also are an indicator for progression under anastrozole [5]. According to two studies in a breast cancer model cell line, the suppression of osteopontin gene expression can enhance radiosensitivity and affect cell apoptosis, suggesting that the molecule may be a target for the improvement of radiotherapy [6,7]. In all these cases, pan-osteopontin was measured. Osteopontin is subject to alternative splicing in cancer, and it is not known which splice form is responsible for conveying resistance to which specific treatment. The variant forms are distinguishable by antibodies to exon 4, recognizing osteopontin-a and osteopontin-b, or to the splice junction of osteopontin-c respectively. Here we test the association of osteopontin splice variants, expressed in the growths at the onset of cancer therapy, with the ensuing response to specific treatments.

\section{Methods}

\section{Patients}

This study contained 119 patients from Poland who presented between 1995 and 2008 (allowing the assessment of 5-year survival). All cases refer to invasive ductal carcinoma, grades 1,2 and 3, with subtypes including few mucinous and tubular carcinomas. Information about the patients was received from the Department of General and Oncological Surgery, Wroclaw and from the Division of Oncological Surgery, Walbrzych, Poland. The inclusion criteria were size of tumor not larger than $50 \mathrm{~mm}$, and no adjuvant chemotherapy at the time of immunohistochemistry. For all patients, who met these criteria, paraffin blocks were available for evaluation. The data comprised also information about pathological TNM (pTNM), BRCA1 status, HER2, ER and PR status, and family history (other cases of invasive breast carcinoma in the family). Ensuing treatment constituted combinations of 1 . hormone therapy with tamoxifen; 2 . chemotherapy with CMF (cyclophosphamide, methotrexate, fluorouracil) 6 courses every 28 days; 3. chemotherapy with AC (cyclophosphamide, doxorubicin) 4 courses every 21 days plus CMF 6 courses every 28 days; 4. radiotherapy to the chest (50 Gy; Mon-Fri 2 Gy) 5. radiotherapy to chest and axilla (50 Gy; Mon-Fri 2 Gy).

\section{Immunohistochemistry}

For each antibody a formalin-fixed and paraffin-embedded biopsy specimen from cancer tissue was cut on a microtome in $5 \mu \mathrm{m}$ slices. The antibodies used in this study, after blocking in $2 \%$ donkey serum, were anti-hOPNc IgY (Gallus Immunotech), and LF161 (Larry Fisher). The IgY antibody recognizes the osteopontin-c splice junction and detects the molecule in immunohistochemistry. It
Table 1 Patient characteristics

\begin{tabular}{|c|c|c|c|}
\hline & & $n$ & $\%$ \\
\hline \multirow[t]{5}{*}{ T } & 0 & 1 & 0.8 \\
\hline & 1 & 59 & 49.6 \\
\hline & 2 & 33 & 27.7 \\
\hline & 3 & 6 & 5.0 \\
\hline & undefined & 20 & 16.8 \\
\hline \multirow[t]{5}{*}{ N } & 0 & 58 & 48.7 \\
\hline & 1 & 14 & 11.8 \\
\hline & 2 & 16 & 13.4 \\
\hline & 3 & 11 & 9.2 \\
\hline & undefined & 20 & 16.8 \\
\hline \multirow[t]{3}{*}{ grade } & 1 & 36 & 30.3 \\
\hline & 2 & 65 & 54.6 \\
\hline & 3 & 18 & 15.1 \\
\hline \multirow[t]{3}{*}{ Her2 } & low & 69 & 58.0 \\
\hline & high & 29 & 24.4 \\
\hline & undefined & 21 & 17.6 \\
\hline \multirow[t]{3}{*}{ PR } & - & 64 & 53.8 \\
\hline & + & 54 & 45.4 \\
\hline & undefined & 1 & 0.8 \\
\hline \multirow[t]{3}{*}{ ER } & - & 59 & 49.6 \\
\hline & + & 59 & 49.6 \\
\hline & undefined & 1 & 0.8 \\
\hline \multirow[t]{3}{*}{ BRCA-1 } & wild type & 52 & 43.7 \\
\hline & mutant & 26 & 21.8 \\
\hline & undefined & 41 & 34.5 \\
\hline \multirow[t]{3}{*}{ familial } & no & 44 & 37.0 \\
\hline & yes & 40 & 33.6 \\
\hline & undefined & 35 & 29.4 \\
\hline \multirow[t]{3}{*}{ chemotherapy } & $\begin{array}{l}\text { AC } 4 \text { courses every } 21 \text { days, CMF } 6 \text { courses } \\
\text { every } 28 \text { days }\end{array}$ & 34 & 28.6 \\
\hline & CMF 6 courses every 28 days & 55 & 46.2 \\
\hline & no & 30 & 25.2 \\
\hline \multirow{3}{*}{$\begin{array}{l}\text { radiation } \\
\text { therapy }\end{array}$} & chest (50 Gy; Mon-Fri 2 Gy) & 41 & 34.5 \\
\hline & chest/axilla (50 Gy; Mon-Fri 2 Gy) & 31 & 26.1 \\
\hline & no & 47 & 39.5 \\
\hline \multirow{2}{*}{$\begin{array}{l}\text { hormone } \\
\text { treatment }\end{array}$} & no & 54 & 45.4 \\
\hline & tamoxifen & 62 & 52.1 \\
\hline
\end{tabular}

The patient populations are described according to diverse clinical variables. CMF cyclophosphamide, methotrexate, fluorouracil, AC cyclophosphamide, doxorubicin

was diluted 1:500 to 1:700. The polyclonal rabbit antibody LF161 for staining selectively exon 4 (present in osteopontin-a and -b) was used at 1:1000. For each antibody, the tissues were scored for intensity (maximum 
Table 2 Marker correlations

\begin{tabular}{|c|c|c|c|c|c|c|}
\hline & & Exon 4 cyt.per. & Exon 4 cyt.int. & OPNc nucl.per. & OPNc nucl.int. & Tumor grade \\
\hline exon 4 & Pearson Correlation & 1 & 0.69115 & 0.41194 & 0.53769 & 0.10199 \\
\hline cyt.per. & $p$-value & & $\leq 0.0001$ & $\leq 0.0001$ & $\leq 0.0001$ & 0.2697 \\
\hline exon 4 & Pearson Correlation & & 1 & 0.31522 & 0.50491 & 0.21883 \\
\hline cyt.int. & $p$-value & & & $\underline{0.0005}$ & $\leq 0.0001$ & 0.0168 \\
\hline OPNC & Pearson Correlation & & & 1 & 0.6636 & 0.0679 \\
\hline nucl.per. & $p$-value & & & & $\leq 0.0001$ & 0.4631 \\
\hline OPNC & Pearson Correlation & & & & 1 & 0.08674 \\
\hline nucl.int. & $p$-value & & & & & 0.3483 \\
\hline tumor grade & Pearson Correlation & & & & & 1 \\
\hline
\end{tabular}

The table shows Pearson correlation coefficients and $p$-values for pairwise comparison of the histopathologic markers (osteopontin-c staining intensity, osteopontin-c percent positivity, exon 4 staining intensity, exon 4 percent positivity) and tumor grade. Statistical significance is indicated by underlining, moderate correlation is shown in bold

intensity of the sample $0,1,2$, or 3 ) and percent positivity $(0,1,2$, or 3$)$, separately for nuclei and cytoplasm. The intensity of staining in immunohistochemistry was evaluated as previously described [8] and the classification criteria of intensity followed a published source [9]. The score was given as 0 points if no staining was observed, 1 for weak, 2 for moderate and 3 points for strong staining. Points were assigned to each case by two pathologists who independently evaluated all microscopic slides and in the rare cases of discrepant initial scores, a final score was agreed on after discussion [8].

\section{Statistics}

All statistical analyses were performed using SAS (North Carolina, USA). Correlations between osteopontin-c and clinicopathological variables were assessed with Pearson's correlation test. Correlation coefficients of 0.1 to 0.3 are considered weak, 0.4-0.6 is moderate, and 0.7-0.9 is strong correlation. A $p$-value of 0.05 or lower indicates statistical significance. The primary methods for addressing the study purposes were Cox hazard models, logistic regression models, and the nonparametric Wilcoxon test. Odds ratios estimate the odds of death for a one-unit increase in the independent variable. Unadjusted odds ratios and
$95 \%$ confidence intervals were calculated to investigate the effects of the components of pathological scores on the odds of death. For survival under hormone therapy or chemotherapy, the biomarkers osteopontin-exon-4 and osteopontin-c were also analyzed in a multiple regression framework to adjust the effect for other covariates. Each model contained either osteopontin-exon-4 or osteopontin-c and each other biomarker (tumor size, lymph node involvement, grade, HER2, Progesterone Receptor, Estrogen Receptor, or BRCA1), added one-ata-time. Cox hazard ratios, $p$-values, and Aikaike Information Criterion (AIC) were used.

\section{Results}

\section{Patient characteristics}

Of 119 patients, 46 women (39\%) died from breast cancer within 5 years while 73 women $(61 \%)$ were alive after this observation period. The average age at the time of immunohistochemistry was 53 years for nonsurvivors and 53 years for survivors. All patients, comprising both subgroups, underwent surgery consisting of either modified radical mastectomy with axillary dissection, or conservative breast surgery with axillary

Table 3 Marker correlations

\begin{tabular}{|c|c|c|c|c|c|c|}
\hline & \multicolumn{2}{|c|}{ Parametric (lognormal) } & \multicolumn{2}{|l|}{ Logrank } & \multicolumn{2}{|l|}{ Wilcoxon } \\
\hline & $\overline{x^{2}}$ & $p$-value & $\overline{x^{2}}$ & $\overline{p \text {-value }}$ & $\overline{x^{2}}$ & $\overline{p \text {-value }}$ \\
\hline exon 4 intensity & 4.82 & $\underline{0.0281}$ & 9.8692 & $\underline{0.0197}$ & 16.3818 & $\underline{0.0009}$ \\
\hline exon 4 high/low & & & 7.9144 & $\underline{0.0049}$ & 15.5494 & $\leq 0.0001$ \\
\hline OPNc intensity & 7.24 & $\leq 0.0001$ & 16.9014 & $\underline{0.0007}$ & 27.5348 & $\leq 0.0001$ \\
\hline OPNc high/low & & & 2.1234 & 0.1438 & 6.4847 & $\underline{0.0109}$ \\
\hline tumor grade & 7.63 & 0.0057 & 11.392 & 0.0098 & 9.5087 & 0.0232 \\
\hline
\end{tabular}

Quantitative multivariable analysis and non-parametric tests for the prediction of survival by the markers under study (osteopontin-c, exon 4 , tumor grade). Used were either the staining levels $0,1,2,3$ (intensity) or the combination of 2 and 3 versus 0 and 1 (high/low) under various model assumptions. Underlined $p$-values are considered significant $(p<0.05)$ 
lymph node dissection and post-operative adjuvant therapy (Table 1).

\section{Immunohistochemistry}

The anti-Osteopontin-exon-4 antibody, which recognizes osteopontin-a and -b, stained selectively the cytoplasm. Most tumors displayed osteopontin-c predominantly in their nuclei. The markers correlated moderately between each other (OPNc nuclear intensity, OPNc nuclear percent positivity, exon 4 cytoplasmic intensity, exon 4 cytoplasmic percent positivity), but in contrast to earlier studies they did not correlate with grade (Table 2). Analysis for the association with survival by the markers under investigation (osteopontin-c, exon 4, tumor grade) reflected them as prognostic for outcome. In addition to analyzing the indicators in their original scale, we dichotomized the immunohistochemical biomarkers into low (0-1) or high (2-3). Only the logrank test for dichotomized osteopontin-c fell short of corroborating significance (Table 3).

\section{Cancer treatment}

The patients were subjected to various combinations of hormone treatment, chemotherapy, and radiation. Hormone treatment was tamoxifen. Chemotherapy comprised one of two regimens, CMF (cyclophosphamide, methotrexate, fluorouracil 6 courses every 28 days) or AC/CMF (cyclophosphamide, doxorubicin, 4 courses every 21 days plus CMF 6 courses every 28 days). Radiotherapy was given either to the chest (50 Gy; Mon-Fri 2 Gy) or to chest and axilla (50 Gy; Mon-Fri 2 Gy). Except for hormone therapy, survival after treatment (chemotherapy yes/no, radiation therapy yes/no) was shorter than survival without treatment. This reflects that more comprehensive therapy was given to patients with more aggressive cancers, which are inherently associated with poor prognoses for survival (Table 4).

\section{Osteopontin variants and specific treatment regimens}

Tamoxifen is a selective estrogen receptor modulator that is used for the treatment of both early and advanced $\mathrm{ER}+$ (estrogen receptor positive) breast cancers in preand post-menopausal women. Kaplan-Meier curves (Fig. 1) suggested a moderate survival benefit from treatment. When comparing hormone-treated to non-hormonetreated patients, low-grade cancers (grade 1) responded better to treatment than high-grade cancers (grade 2-3). In contrast, patients with high intensity staining (2-3) of exon 4 or osteopontin-c responded better to hormone therapy than those with low intensity staining $(0-1)$ of these markers, as judged by a divergence with time between the hormone-treated and the non-hormone-treated patient groups at high marker intensity, but much less at low marker intensity.
Table 4 Survival under treatment

\begin{tabular}{|c|c|c|c|}
\hline \multirow[b]{2}{*}{ Treatment } & \multicolumn{3}{|c|}{ Years of survival } \\
\hline & Mean & std & $\mathrm{n}$ \\
\hline hormone no (all subgroups) & 6.48 & 3.96 & 54 \\
\hline hormone yes (all subgroups) & 7.44 & 4.35 & 64 \\
\hline hormone alone & 9.89 & 2.00 & 18 \\
\hline hormone and chemo & 6.63 & 4.38 & 16 \\
\hline hormone and radiation & 5.10 & 4.63 & 10 \\
\hline hormone, chemo, radiation & 7.10 & 4.84 & 21 \\
\hline chemo no (all subgroups) & 8.30 & 3.79 & 30 \\
\hline chemo yes (all subgroups) & 6.57 & 4.22 & 89 \\
\hline chemo alone & 7.92 & 4.57 & 13 \\
\hline chemo and hormone & 6.63 & 4.38 & 16 \\
\hline chemo and radiation & 5.82 & 3.66 & 39 \\
\hline chemo, hormone, radiation & 7.10 & 4.84 & 21 \\
\hline chemo (CMF 6 courses every 28 days) & 6.27 & 4.36 & 55 \\
\hline $\begin{array}{l}\text { chemo (AC } 4 \text { courses every } 21 \text { days, } \\
\text { CMF } 6 \text { courses every } 28 \text { days) }\end{array}$ & 7.06 & 4.00 & 34 \\
\hline radiation no (all subgroups) & 8.23 & 3.90 & 47 \\
\hline radiation yes (all subgroups) & 6.21 & 4.17 & 72 \\
\hline radiotherapy alone & 10.00 & 1.41 & 2 \\
\hline radiation and hormone & 5.10 & 4.63 & 10 \\
\hline radiation and chemo & 5.82 & 3.66 & 39 \\
\hline radiation, chemo, hormone & 7.10 & 4.84 & 21 \\
\hline
\end{tabular}

Shown are mean survival times in years (mean), standard deviation (std), and number of cases ( $n$ ) consecutive to the various combinations of treatment. When censored for 13-year survivors (who may be alive beyond 13 years), the mean survival of patients under hormone treatment is 6.423 years with standard error 0.5274 , compared to survival under no hormone treatment of 7.262 years and standard error 0.5077

For the analysis of chemotherapy responses, the comparison to the non-chemotherapy treated group was not meaningful, because the survival of the treated group was lower, which is not reflecting harm caused by the treatment but indicates the circumstance that chemotherapy was given to patients with aggressive tumors and poor prognoses. The survival curves of patients undergoing chemotherapy (Fig. 2), when distinguished according to low (0-1) versus high (2-3) immunohistochemical markers, confirm the poor survival prognosis associated with exon 4 and osteopontin-c [8], particularly reflected in a higher rate of patient deaths between 2 and 6 years after diagnosis in the high intensity group of each marker. Of note, the survival difference between exon 4 high and low appeared to be larger than between osteopontin-c high and low, implying the possibility that the marker could also be predictive of a poor chemotherapy response.

The Kaplan Meier survival curves of patients undergoing radiation treatment, distinguished according to low $(0-1)$ versus high $(2-3)$ immunohistochemical markers, 
Osteopontin-c
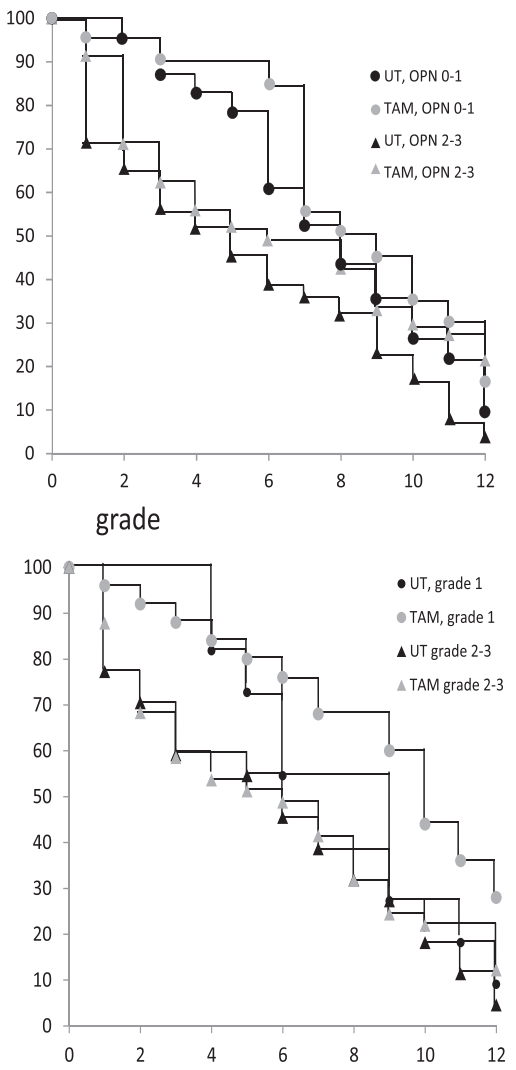

Exon 4
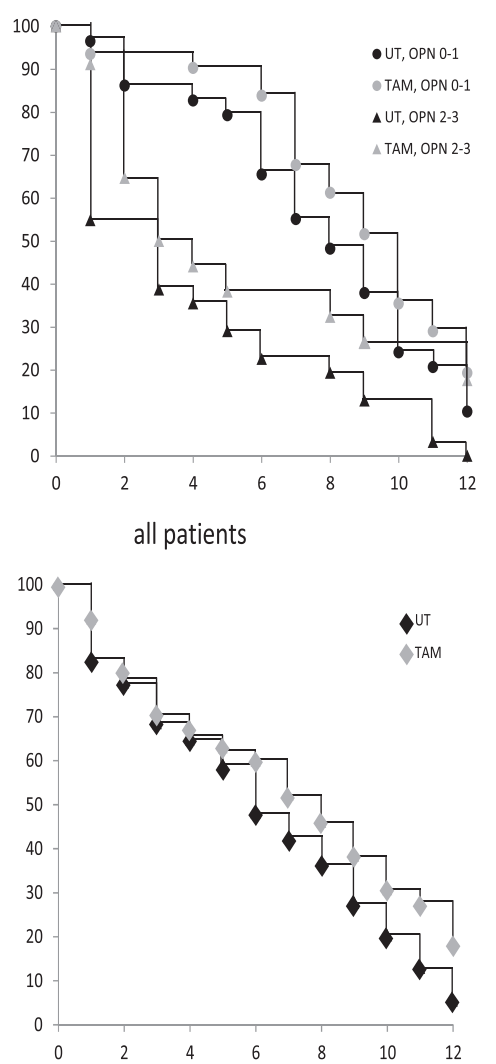

Fig. 1 Kaplan-Meier survival curves for patients undergoing hormone therapy. Survival of patients under hormone treatment, distinguished according to low (levels $0-1$ ) versus high (levels 2-3) osteopontin-c staining (top left), low versus high osteopontin-exon 4 staining (top right), or low (1) versus high (2-3) tumor grade (bottom left). The overall survival of patients receiving hormone treatment versus not receiving hormone treatment is shown for comparison on the bottom right. Untreated subgroups are displayed in black, treated subgroups in gray, low marker levels are shown as circles, high marker levels as triangles. The $x$-axis indicates years since diagnosis, the $y$-axis reflects \% surviving patients

again confirm the poor survival associated with exon 4 and osteopontin-c. Although the curves converge after about 10 years, during 2-6 years substantially more patients die in the high marker intensity groups than in the low marker intensity groups (Fig. 3).

\section{Osteopontin variants as predictive markers}

The above findings (reduced survival of high versus low exon 4 and osteopontin-c under chemotherapy or radiation therapy) can be interpreted as poor therapy responses only if they are a) significant and b) distinct from the overall prognostic nature of these markers, as previously reported $[8,10,11]$. The seemingly favorable survival of high versus low exon 4 and osteopontin-c under hormone therapy is distinct from the prognostic characteristic, but requires testing for significance [12].

Cox hazard ratios (Table 5) showed that the staining intensity for osteopontin exon 4 was negatively correlated with survival in the non-hormone treated group, but not in the hormone treated group. This is consistent with a favorable response to tamoxifen associated with the presence of osteopontin exon 4. By contrast, osteopontin exon 4 was associated with a poor response to chemotherapy according to reduced survival by high level expressors in the treated group, but not in the nonchemotherapy treated group. CMF (not AC/CMF) was the regimen, the efficacy of which was compromised by high osteopontin exon 4. Because a supremum test indicated low confidence in the proportionality assumption, we sought to independently corroborate the analysis using the logrank and Wilcoxon tests (Table 6). According to all 3 tests, exon 4 is predictive of resistance to chemotherapy with CMF (as judged by significantly worse survival of the high marker intensity group), but not with AC/CMF. According to Cox hazard ratios and logrank test, exon 4 is a predictor of a favorable response to hormone therapy (the significantly worse survival associated with high marker intensity in the non-hormone treated group is lost under hormone therapy with tamoxifen). Although a similar predictive potential (for a favorable 


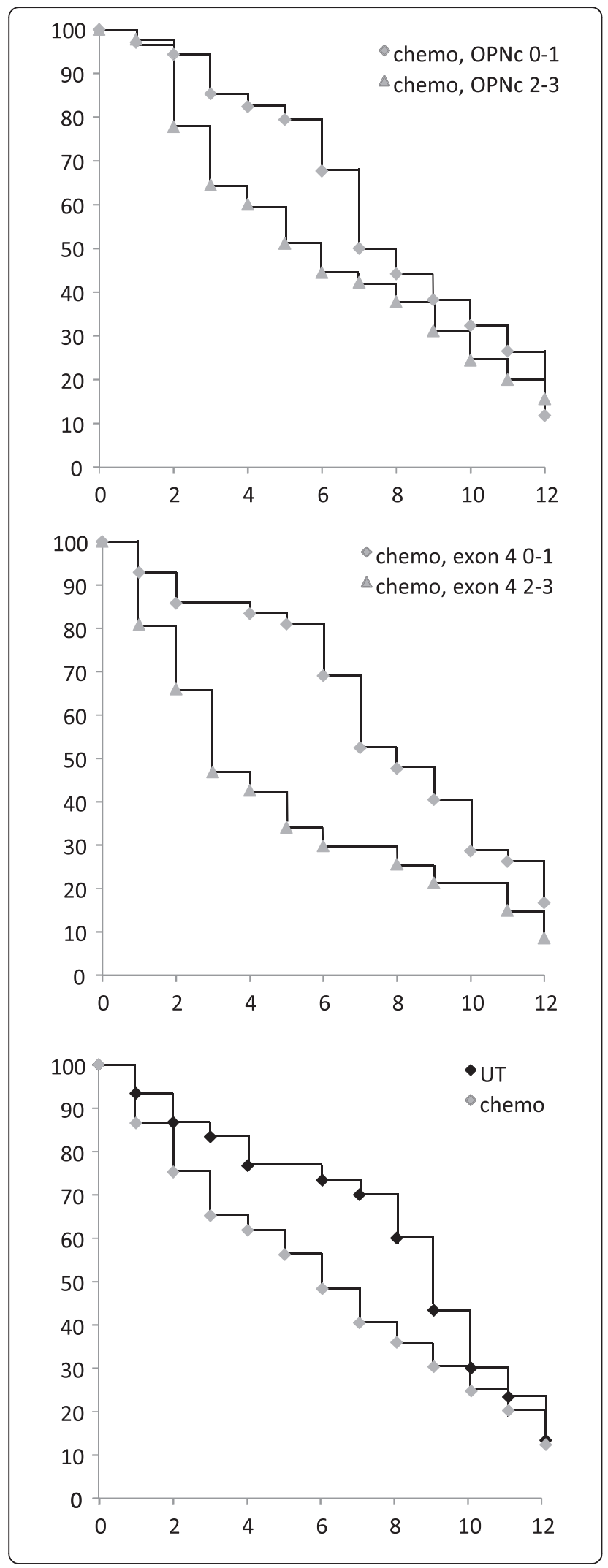

Fig. 2 Kaplan-Meier survival curves for patients undergoing chemotherapy. Survival of patients under chemotherapy, distinguished according to low (0-1, diamonds) versus high (2-3, triangles) immunohistochemical markers. Shown are Kaplan Meier curves for osteopontin-c (top panel) or exon 4 (middle panel). For comparison, the survival of all patients treated (gray markers) or not treated (black markers) with chemotherapy is displayed (bottom panel). The $x$-axis indicates years since diagnosis, the $y$-axis reflects \% surviving patients

response to hormone therapy) is implied for osteopontin-c, the $p$-values for the Cox and logrank tests in the "hormone no" group fall just short of statistical significance.

To assess whether the prediction of treatment responses can be strengthened when additional readouts are considered, we performed multivariate analysis. For survival with or without hormone therapy, exon 4 staining or osteopontin-c staining were analyzed as predictors in conjunction with other covariates (Table 7). Whereas osteopontin-c did not improve as a predictor, the combination of high exon 4 intensity plus high tumor grade worsened the prognosis without hormone therapy (exon 4 alone hazard ratio 0.503, $p$-value 0.016 ; with grade 0.450 , 0.007 ), but maintains the favorable prognosis under treatment (exon 4 alone $0.657,0.131$; with grade $0.763,0.352$ ). Hazard ratios and $p$-values for survival under CMF chemotherapy showed improvement for the prediction with the combination of exon 4 intensity plus HER2 (0.398, 0.009), compared to exon 4 intensity alone $(0.524,0.019)$. Low HER2 and high staining intensity for osteopontin exon 4 increase the likelihood for resistance to CMF chemotherapy (Table 8).

\section{Discussion}

In this study, we have identified osteopontin exon 4 as a predictor for a favorable treatment response to tamoxifen (the staining intensity is negatively correlated with survival in the non-hormone treated group, but not in the hormone treated group), but for resistance to chemotherapy with CMF (high level expressors have reduced survival in the treated group, but not in the non-treated group). The combination of high staining for osteopontin exon 4 with HER2 negative status appears to increase the likelihood for chemotherapy resistance. By contrast, osteopontin-c is prognostic, but may not be predictive for the therapeutic regimens applied here. Notably, while osteopontin-c is not a predictor of survival in the hormone treated group, a trend for it to be negatively associated with survival time in the non-hormone treated group fell just short of indicating significance by Cox hazard ratio $(p=0.096)$ and logrank test $(p=0.066)$, while significance was attained with the Wilcoxon test $(p=0.021)$. Nevertheless, in multivariate analysis no improvement was achieved for prediction with osteopontin-c. It is therefore more likely that a favorable response to 


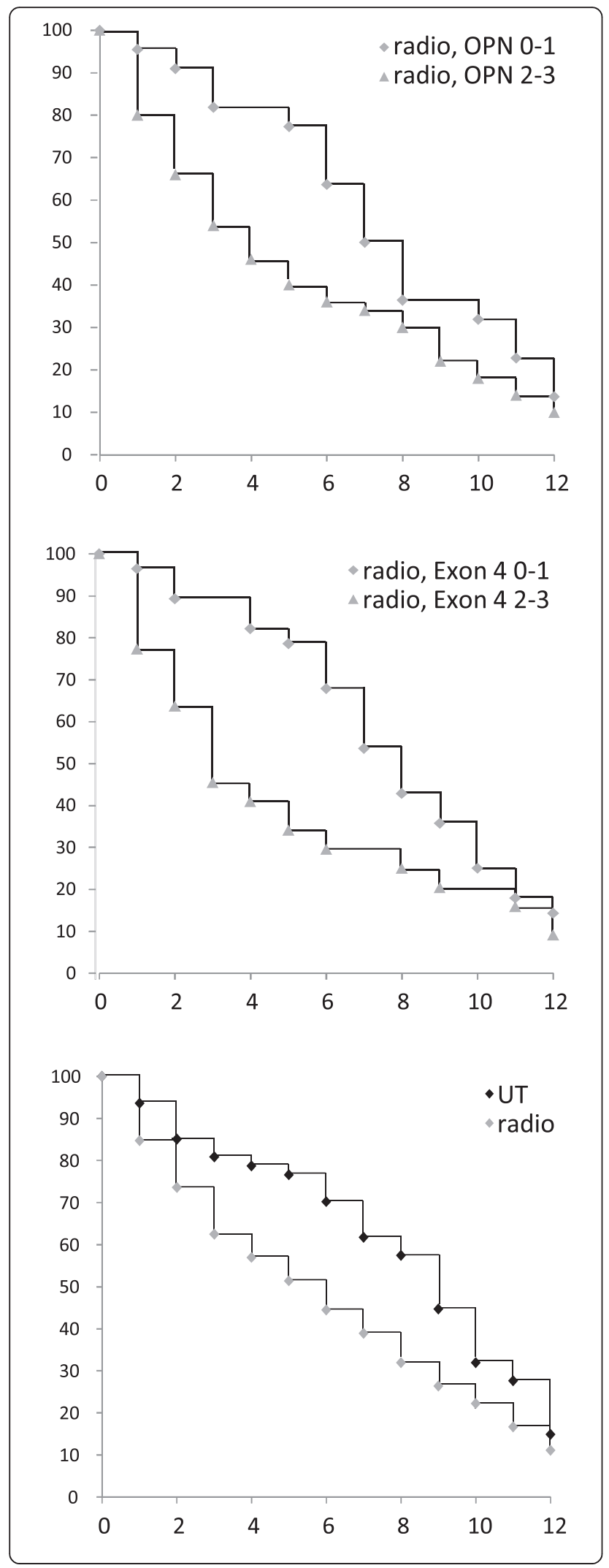

Fig. 3 Kaplan-Meier survival curves for patients undergoing radiotherapy. Survival of patients under radiotherapy, distinguished according to low ( $0-1$, diamonds) versus high ( $2-3$, triangles) immunohistochemical markers. Shown are Kaplan Meier curves for osteopontin-c (top panel) or exon 4 (middle panel). For comparison, the survival of all patients receiving radiation (gray markers) or not treated with radiation (black markers) is displayed (bottom panel). The $x$-axis indicates years since diagnosis, the $y$-axis reflects \% surviving patients

hormone therapy is encoded in an osteopontin-a specific domain than in a common domain of osteopontin. The resistance to chemotherapy is selective to exon 4. As osteopontin-b is barely expressed in breast cancer and the protein is rapidly degraded [13], it is implied that osteopontin-a is the splice form responsible for resistance to chemotherapy.

The favorable response to anti-estrogens by breast cancers with high osteopontin-a levels may reflect the property of the osteopontin gene as a sentinel for estrogen responsiveness in mammary cells. Although an estrogenresponse element is not present in the promoter, there are 7 steroid factor-response element-like sequences in this region. Expression may be induced by estrogen via ERR $\alpha$ in a context-dependent manner [14, 15]. Tumors with high levels of osteopontin-a probably are highly sensitive to estrogen signals (mediated through ER or through ERR), and therefore will be more likely to be susceptible to tamoxifen treatment. In immunohistochemistry, the abundance of osteopontin may be more accurately reflected in staining intensity than in percent positivity, because the percentage of area stained is much more susceptible to the placement of the section than is the intensity, making the former a weaker readout. In a related setting, for ER in breast cancer, it has been shown that the threshold of immunoreactivity is more important than the percentage positive in the generation of discordant or false-negative assays [16].

The abundance of exon 4 is predictive of resistance to chemotherapy with CMF, but not with AC/CMF. This was initially surprising as osteopontin was not previously known to convey resistance to methotrexate or fluorouracil. In contrast, the two AC agents, doxorubicin (adriamycin) and cyclophosphamide, have been reported to be subject to osteopontin-mediated drug resistance. The finding may point to translational limitations for testing drug sensitivity with mono-therapy in cell culture, as was done in the published resistance studies [1-4]. It may also be reflective of the benefit conveyed with altering drug combinations over treatment cycles, not only to alleviate toxicity but also to enhance efficacy. By broadening and alternating the drug regimen, the response to treatment in our breast cancer patients could be enhanced. 
Table 5 Survival under specific treatments

\begin{tabular}{|c|c|c|c|c|c|c|c|c|c|c|c|c|c|c|c|c|c|}
\hline \multirow[b]{2}{*}{ Treatment } & \multirow[b]{2}{*}{$\mathrm{n}$} & \multicolumn{4}{|c|}{ Exon 4 intensity } & \multicolumn{4}{|c|}{ Exon $4 \%$} & \multicolumn{4}{|c|}{ OPN-c intensity } & \multicolumn{4}{|c|}{ OPN-c \% } \\
\hline & & $\begin{array}{l}\text { Odds } \\
\text { ratio }\end{array}$ & $95 \% \mathrm{Cl}$ & $p$-value & $\begin{array}{l}\text { Supre-mum } \\
\text { test }\end{array}$ & $\begin{array}{l}\text { Odds } \\
\text { ratio }\end{array}$ & $95 \% \mathrm{Cl}$ & $p$-value & $\begin{array}{l}\text { Supre-mum } \\
\text { test }\end{array}$ & $\begin{array}{l}\text { Odds } \\
\text { ratio }\end{array}$ & $95 \% \mathrm{Cl}$ & $p$-value & $\begin{array}{l}\text { Supre-mum } \\
\text { test }\end{array}$ & $\begin{array}{l}\text { Odds } \\
\text { ratio }\end{array}$ & $95 \% \mathrm{Cl}$ & $p$-value & $\begin{array}{l}\text { Supre-mum } \\
\text { test }\end{array}$ \\
\hline $\begin{array}{l}\text { hormone no } \\
\text { (all subgroups) }\end{array}$ & 54 & 0.503 & $0.287-0.881$ & 0.016 & 0.104 & 1.087 & $0.624-1.895$ & 0.767 & 0.908 & 0.620 & $0.353-1.089$ & 0.096 & 0.178 & 1.210 & $0.696-2.102$ & 0.500 & 0.954 \\
\hline $\begin{array}{l}\text { hormone yes } \\
\text { (all subgroups) }\end{array}$ & 65 & 0.657 & $0.381-1.134$ & 0.131 & $<.0001$ & 0.687 & $0.391-1.206$ & 0.191 & 0.056 & 0.826 & $0.463-1.473$ & 0.517 & 0.005 & 1.101 & $0.630-1.923$ & 0.735 & 0.704 \\
\hline hormone alone & 18 & 1.534 & $0.430-5.467$ & 0.509 & 0.614 & 1.122 & $0.420-2.998$ & 0.819 & 0.616 & 1.661 & $0.607-4.542$ & 0.323 & 0.518 & 1.113 & $0.383-3.235$ & 0.845 & 0.607 \\
\hline $\begin{array}{l}\text { hormone and } \\
\text { chemo }\end{array}$ & 16 & 0.412 & $0.129-1.316$ & 0.134 & 0.022 & 0.637 & $0.173-2.343$ & 0.497 & 0.064 & 0.634 & $0.204-1.973$ & 0.432 & 0.049 & 1.352 & $0.441-4.148$ & 0.598 & 0.269 \\
\hline $\begin{array}{l}\text { hormone and } \\
\text { radiation }\end{array}$ & 10 & 0.358 & $0.069-1.862$ & 0.222 & 0.180 & 0.495 & $0.096-2.558$ & 0.401 & 0.568 & 0.250 & $0.029-2.180$ & 0.209 & 0.185 & 0.827 & $0.192-3.561$ & 0.798 & 0.597 \\
\hline $\begin{array}{l}\text { hormone, } \\
\text { chemo, radiation }\end{array}$ & 21 & 0.598 & $0.169-2.112$ & 0.424 & 0.763 & 0.604 & $0.218-1.676$ & 0.333 & 0.801 & 0.867 & $0.278-2.710$ & 0.806 & 0.375 & 0.894 & $0.310-2.580$ & 0.836 & 0.810 \\
\hline $\begin{array}{l}\text { chemo no } \\
\text { (all subgroups) }\end{array}$ & 30 & 0.711 & $0.320-1.580$ & 0.403 & 0.025 & 0.794 & $0.366-1.722$ & 0.560 & 0.233 & 0.903 & $0.391-2.084$ & 0.811 & 0.134 & 0.978 & $0.425-2.254$ & 0.959 & 0.612 \\
\hline $\begin{array}{l}\text { chemo yes } \\
\text { (all subgroups) }\end{array}$ & 89 & 0.582 & $0.371-0.913$ & $\underline{0.019}$ & 0.001 & 0.873 & $0.552-1.383$ & 0.564 & 0.520 & 0.694 & $0.439-1.098$ & 0.119 & 0.003 & 1.181 & $0.754-1.848$ & 0.468 & 0.327 \\
\hline chemo (CMF) & 55 & 0.524 & $0.290-0.943$ & $\underline{0.031}$ & 0.008 & 0.727 & $0.403-1.314$ & 0.291 & 0.693 & 0.706 & $0.391-1.276$ & 0.249 & 0.013 & 1.113 & $0.626-1.979$ & 0.715 & 0.337 \\
\hline chemo (AC/CMF) & 34 & 0.688 & $0.338-1.400$ & 0.302 & 0.144 & 1.252 & $0.596-2.632$ & 0.553 & 0.543 & 0.681 & $0.329-1.410$ & 0.301 & 0.121 & 1.280 & $0.608-2.694$ & 0.515 & 0.727 \\
\hline chemo alone & 13 & 0.418 & $0.111-1.574$ & 0.197 & 0.763 & 1.207 & $0.367-3.971$ & 0.757 & 0.637 & 0.711 & $0.205-2.468$ & 0.592 & 0.047 & 1.200 & $0.362-3.980$ & 0.766 & 0.560 \\
\hline $\begin{array}{l}\text { chemo and } \\
\text { radiation }\end{array}$ & 39 & 0.546 & $0.285-1.047$ & 0.069 & 0.027 & 1.343 & $0.686-2.629$ & 0.389 & 0.449 & 0.623 & $0.320-1.213$ & 0.164 & 0.444 & 1.215 & $0.641-2.304$ & 0.551 & 0.950 \\
\hline $\begin{array}{l}\text { radiation no } \\
\text { (all subgroups) }\end{array}$ & 47 & 0.647 & $0.333-1.259$ & 0.200 & 0.024 & 0.973 & $0.519-1.824$ & 0.933 & 0.203 & 0.981 & $0.527-1.826$ & 0.951 & 0.105 & 1.400 & $0.746-2.627$ & 0.294 & 0.404 \\
\hline $\begin{array}{l}\text { radiation yes } \\
\text { (all subgroups) }\end{array}$ & 72 & 0.626 & $0.376-1.044$ & 0.073 & 0.002 & 0.774 & $0.466-1.285$ & 0.321 & 0.489 & 0.675 & $0.394-1.157$ & 0.153 & 0.036 & 1.086 & $0.659-1.791$ & 0.745 & 0.832 \\
\hline
\end{tabular}


Table 6 Survival under specific treatments

\begin{tabular}{|c|c|c|c|c|c|c|c|c|c|c|c|c|c|c|c|c|c|}
\hline \multirow[b]{3}{*}{ Treatment } & \multirow[b]{3}{*}{$n$} & \multicolumn{4}{|c|}{ Exon 4 intensity } & \multicolumn{4}{|c|}{ Exon 4 \% } & \multicolumn{4}{|c|}{ OPN-c intensity } & \multicolumn{4}{|c|}{ OPN-c \% } \\
\hline & & \multicolumn{2}{|c|}{ Logrank } & \multicolumn{2}{|c|}{ Wilcoxon } & \multicolumn{2}{|c|}{ Logrank } & \multicolumn{2}{|c|}{ Wilcoxon } & \multicolumn{2}{|c|}{ Logrank } & \multicolumn{2}{|c|}{ Wilcoxon } & \multicolumn{2}{|c|}{ Logrank } & \multicolumn{2}{|c|}{ Wilcoxon } \\
\hline & & $x^{2}$ & $\overline{p \text {-value }}$ & $\overline{x^{2}}$ & $\overline{p \text {-value }}$ & $\overline{x^{2}}$ & $\overline{p \text {-value }}$ & $x^{2}$ & $\overline{p \text {-value }}$ & $\overline{x^{2}}$ & $\overline{p \text {-value }}$ & $x^{2}$ & $\overline{p \text {-value }}$ & $x^{2}$ & $\overline{p \text {-value }}$ & $x^{2}$ & $\overline{p \text {-value }}$ \\
\hline hormone no (all subgroups) & 54 & 7.127 & $\underline{0.008}$ & 9.087 & 0.003 & 0.106 & 0.745 & 0.015 & 0.902 & 3.384 & 0.066 & 5.373 & $\underline{0.021}$ & 0.551 & 0.458 & 0.372 & 0.542 \\
\hline hormone yes (all subgroups) & 65 & 2.631 & 0.105 & 6.981 & 0.008 & 1.970 & 0.160 & 3.267 & 0.071 & 0.480 & 0.489 & 2.218 & 0.136 & 0.130 & 0.719 & 0.140 & 0.708 \\
\hline hormone alone & 18 & 0.591 & 0.442 & 0.226 & 0.635 & 0.069 & 0.793 & 0.071 & 0.790 & 1.303 & 0.254 & 0.961 & 0.327 & 0.050 & 0.823 & 0.184 & 0.668 \\
\hline hormone and chemo & 16 & 2.613 & 0.106 & 3.937 & 0.047 & 0.531 & 0.466 & 1.071 & 0.301 & 0.707 & 0.401 & 1.778 & 0.182 & 0.317 & 0.573 & 0.101 & 0.751 \\
\hline hormone and radiation & 10 & 1.833 & 0.176 & 2.709 & 0.100 & 0.837 & 0.360 & 0.987 & 0.320 & 2.049 & 0.152 & 2.485 & 0.115 & 0.075 & 0.784 & 0.011 & 0.915 \\
\hline hormone, chemo, radiation & 21 & 0.703 & 0.402 & 0.601 & 0.438 & 1.036 & 0.309 & 1.139 & 0.286 & 0.065 & 0.799 & 0.240 & 0.624 & 0.047 & 0.829 & 0.089 & 0.765 \\
\hline chemo no (all subgroups) & 30 & 0.833 & 0.361 & 3.472 & 0.062 & 0.403 & 0.526 & 1.123 & 0.289 & 0.067 & 0.795 & 0.465 & 0.495 & 0.003 & 0.955 & 0.049 & 0.825 \\
\hline chemo yes (all subgroups) & 89 & 6.588 & $\underline{0.010}$ & 10.593 & 0.001 & 0.387 & 0.534 & 0.566 & 0.452 & 2.858 & 0.091 & 7.465 & $\underline{0.006}$ & 0.614 & 0.433 & 0.186 & 0.667 \\
\hline chemo (CMF) & 55 & 5.455 & $\underline{0.020}$ & 9.345 & 0.002 & 1.275 & 0.259 & 1.561 & 0.212 & 1.522 & 0.217 & 4.847 & $\underline{0.028}$ & 0.152 & 0.697 & 0.016 & 0.899 \\
\hline chemo (AC/CMF) & 34 & 1.289 & 0.256 & 1.608 & 0.205 & 0.420 & 0.517 & 0.317 & 0.574 & 1.300 & 0.254 & 2.740 & 0.098 & 0.507 & 0.477 & 0.456 & 0.500 \\
\hline chemo alone & 13 & 1.865 & 0.172 & 1.268 & 0.260 & 0.107 & 0.743 & 0.245 & 0.620 & 0.322 & 0.570 & 1.356 & 0.244 & 0.099 & 0.753 & 0.126 & 0.722 \\
\hline chemo and radiation & 39 & 4.123 & $\underline{0.042}$ & 8.229 & $\underline{0.004}$ & 0.898 & 0.344 & 0.209 & 0.648 & 2.380 & 0.123 & 3.271 & 0.071 & 0.431 & 0.512 & 0.180 & 0.672 \\
\hline radiation no (all subgroups) & 47 & 1.964 & 0.161 & 3.638 & 0.057 & 0.009 & 0.927 & 0.067 & 0.796 & 0.005 & 0.946 & 0.497 & 0.481 & 1.310 & 0.252 & 1.819 & 0.178 \\
\hline radiation yes (all subgroups) & 72 & 3.786 & 0.052 & 8.444 & 0.004 & 1.143 & 0.285 & 1.690 & 0.194 & 2.395 & 0.122 & 4.766 & 0.029 & 0.122 & 0.727 & 0.090 & 0.764 \\
\hline
\end{tabular}


Table 7 Multivariate analysis

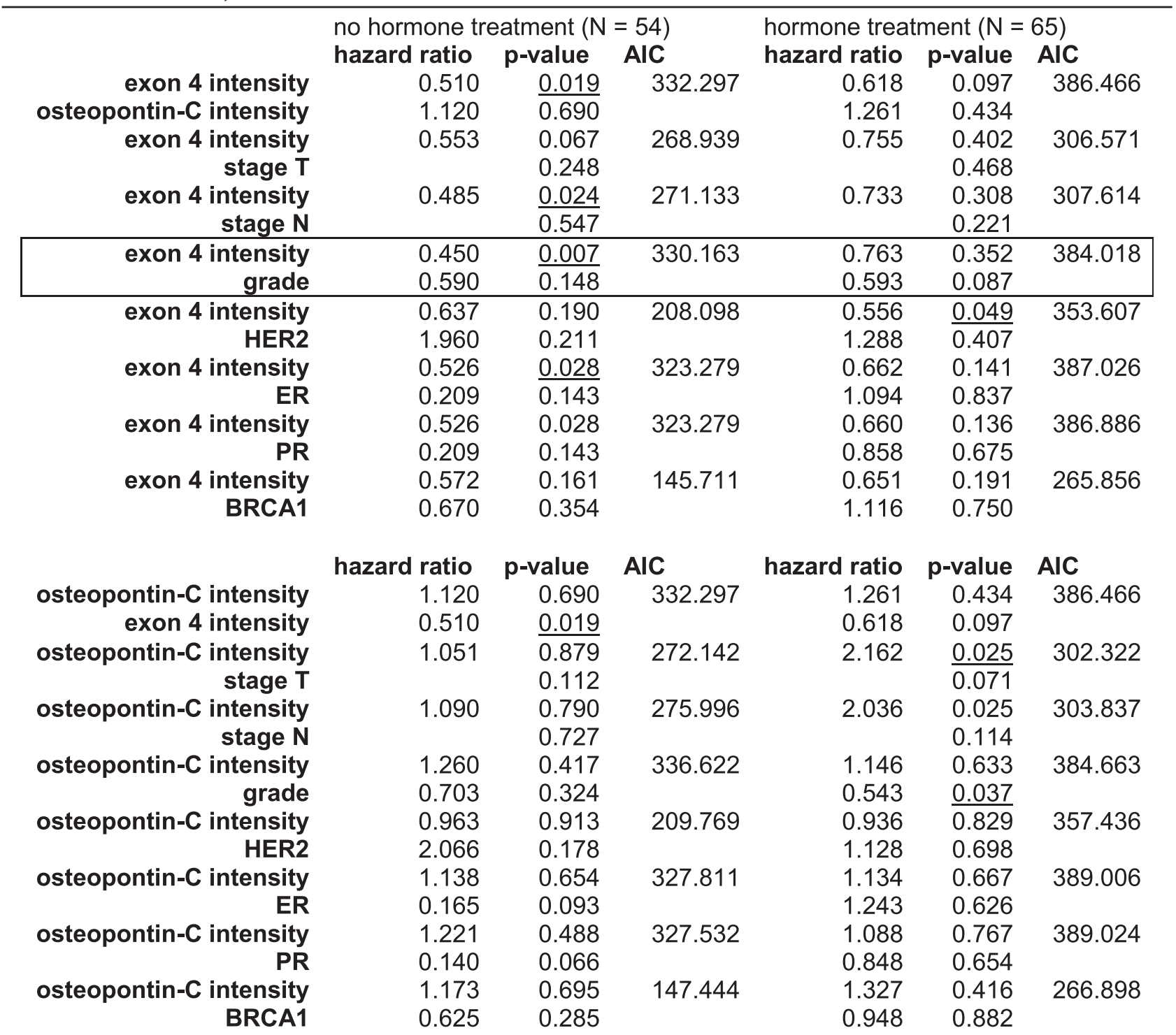

Hazard ratios and $p$-values for survival under hormone therapy, using exon 4 staining $(0-1=$ low, $2-3=$ high) or osteopontin-c staining (0-1 = low, $2-3=$ high) plus other readouts as covariates. The combination of exon 4 intensity plus grade, which strengthens the prediction compared to exon 4 intensity alone, is boxed

Discrepancies between this study and previous reports that had indicated an association between osteopontin levels and radiation resistance require an explanation. We see three likely causes. a) While the regimens tested here had overlap with the ones earlier associated with resistance, the treatments were complex and included components that may have helped to overcome resistance. Most patients in this study received radiation in conjunction with hormone therapy and/or chemotherapy. b) Overall, the efficacies of radiation treatment were low, reflected in poor survival in the treated groups. The underlying reason is that more aggressive treatment is given to patients with worse prognosis and could be interpreted to mean that all patients receiving radiation were in essence resistant. Therefore, no differences were discernible between the marker (osteopontin-c or exon 4) high and low groups. c) It is possible that the observations of this study may be compromised by its moderate power (119 patients). We previously reported that osteopontin-c is correlated to tumor grade [13] and the combination of both readouts slightly improves the prediction of survival [8]. In the present study, no correlation was identified between osteopontin-c and tumor grade or exon 4 and tumor grade, which may be attributable to the limited group size. The lack of significance for predicting survival by dichotomized osteopontin-c in the logrank test might seem to support this possibility. 
Table 8 Multivariate analysis

\begin{tabular}{|c|c|c|c|c|c|c|c|c|c|c|c|c|}
\hline & \multicolumn{3}{|c|}{ chemo $N(N=30)$} & \multicolumn{3}{|c|}{ chemo $Y(N=89)$} & \multicolumn{3}{|c|}{ chemo CMF $(\mathrm{N}=55)$} & \multicolumn{3}{|c|}{ chemo $\mathrm{AC} / \mathrm{CMF}(\mathrm{N}=34)$} \\
\hline & $\begin{array}{l}\text { hazard } \\
\text { ratio }\end{array}$ & $p$-value & AIC & $\begin{array}{l}\text { hazard } \\
\text { ratio }\end{array}$ & p-value & AIC & $\begin{array}{l}\text { hazard } \\
\text { ratio }\end{array}$ & p-value & AIC & $\begin{array}{l}\text { hazard } \\
\text { ratio }\end{array}$ & p-value & AIC \\
\hline exon 4 intensity & 0.710 & 0.403 & 150.252 & 0.561 & $\underline{0.013}$ & 600.877 & 0.517 & $\underline{0.028}$ & 320.120 & 0.645 & 0.235 & 181.221 \\
\hline osteopontin-C intensity & 1.016 & 0.971 & & 1.286 & $\overline{0.276}$ & & 1.172 & $\overline{0.591}$ & & 1.407 & 0.377 & \\
\hline $\begin{array}{r}\text { stage T } \\
\text { exon } 4 \text { intensity }\end{array}$ & 0.845 & $\begin{array}{l}0.266 \\
0.736\end{array}$ & 110661 & 0.633 & $\begin{array}{l}0.226 \\
0.067\end{array}$ & 492113 & 0.556 & $\begin{array}{l}0.108 \\
0.072\end{array}$ & 253,430 & 0.741 & 0.872 & 157329 \\
\hline $\begin{array}{r}\text { exon } 4 \text { intensity } \\
\text { stage } N\end{array}$ & & $\underline{0.018}$ & & 0.033 & 0.942 & & & 0.884 & & 0.141 & 0.884 & \\
\hline exon 4 intensity & 0.743 & $\overline{0.467}$ & 149.228 & 0.605 & $\underline{0.030}$ & 597.491 & 0.560 & 0.059 & 319.493 & 0.604 & 0.177 & 177.012 \\
\hline exon 4 intensity & 0.714 & 0.418 & 143.092 & 0.512 & $\underline{0.012}$ & 430.832 & 0.398 & $\underline{0.009}$ & 224.108 & 0.671 & 0.333 & 127.586 \\
\hline HER2 & 1.086 & 0.849 & & 1.681 & $\overline{0.095}$ & & 2.751 & $\underline{\underline{0.029}}$ & & 1.000 & 1.000 & \\
\hline exon 4 intensity & 0.712 & 0.403 & 150.246 & 0.563 & $\underline{0.014}$ & 591.012 & 0.511 & $\underline{0.027}$ & 311.047 & 0.664 & 0.264 & 181.498 \\
\hline ER & 1.043 & 0.933 & & 1.420 & $\overline{0.148}$ & & 1.486 & $\overline{0.224}$ & & 1.288 & 0.492 & \\
\hline exon 4 intensity & 0.700 & 0.383 & 150.005 & 0.554 & $\underline{0.012}$ & 591.544 & 0.511 & $\underline{0.028}$ & 311.932 & 0.639 & 0.227 & 181.122 \\
\hline PR & 0.795 & 0.625 & & 1.368 & 0.209 & & 1.300 & 0.424 & & 1.422 & 0.362 & \\
\hline exon 4 intensity & 0.722 & 0.466 & 115.922 & 0.770 & 0.113 & 306.298 & 0.487 & 0.085 & 135.509 & 0.860 & 0.743 & 110.253 \\
\hline BRCA1 & 1.084 & 0.864 & & 0.909 & 0.770 & & 1.617 & 0.386 & & 0.502 & 0.145 & \\
\hline
\end{tabular}

Hazard ratios and $p$-values for survival under chemotherapy, using exon 4 staining (0-1 = low, 2-3= high) plus other readouts as covariates. The combination of exon 4 intensity plus HER2, which strengthens the prediction compared to exon 4 intensity alone, is boxed. Tumor grade was included as low (grade 1) or high (grades 2-3). The parameters for tumor size and lymph node involvement, were not dichotomized and had different hazard ratios for each level (not shown). Significant $p$-values $(p<0.05)$ are underlined

Beside breast cancer, pan-osteopontin levels have been associated with treatment responses of prostate cancer to taxanes/androgen deprivation [17], of lung cancer (NSCLC) to carboplatin/paclitaxel [18], and of colorectal cancer to FOLFIRI-bevacizumab [19] (as osteopontin interacts with VEGF in multiple ways [20] it would be expected to affect the response to bevacizumab). In some of the predecessor studies, however, it has not been clear whether osteopontin was functionally linked to a reduced treatment response or independently predicted poor survival, because tumors with higher levels of the biomarker are more aggressive and inherently have a bad prognosis. A mechanistic connection of pan-osteopontin to drug resistance was established in prostate cancer. Osteopontin binds to integrin $\alpha_{\mathrm{v}} \beta_{3}$ and concentrationand time-dependently upregulates the efflux transporter ABCB1 (P-glycoprotein, PGP), which causes resistance to drugs that are substrates for this transporter [21]. Similarly in breast tumor cells, osteopontin may activate the hedgehog pathway and enhance drug resistance through GLI1dependent regulation of ABCB1 and ABCG2 [4].

\section{Conclusions}

In conclusion, immunohistochemistry of osteopontin splice variants has potential to aid decision making in breast cancer treatment. Specifically, osteopontin exon 4 is associated with a favorable response to tamoxifen, but a poor response to chemotherapy with CMF (cyclophosphamide, methotrexate, fluorouracil). While being prognostic, osteopontin-c may not be a significant predictor for sensitivity to treatment.

\section{Abbreviations}

AC, cyclophosphamide, doxorubicin; AIC, Aikaike information criterion; CMF, cyclophosphamide, methotrexate, fluorouracil; ER, estrogen receptor; OPN, osteopontin; PR, progesterone receptor; sem, standard error of the mean; Std, standard deviation

\section{Acknowledgements}

We are grateful to Dr. Larry Fisher, $\mathrm{NIH}$, for having generously provided the antibody LF161 (anti-Osteopontin-exon-4). Biostatistics support was provided through the University of Cincinnati CCTST (Center for Clinical \& Translational Science \& Training).

\section{Funding}

This research was supported by U.S. DOD grant BC095225 and the Marlene Harris-Ride Cincinnati/Pilot Program to GFW.

Availability of data and materials

Data and Materials can be requested from the authors (identifying patient data will not be shared). Deposition in publically available repositories is not applicable.

\section{Authors' contributions}

KZ: histopathology; AA: specimen collection, provision of patient data; SA: specimen collection, provision of patient data; $\mathrm{MdMH}$ : biostatistics, manuscript preparation; PZ: histopathology, manuscript preparation; GFW: project design, method validation, manuscript preparation. All authors read and approved the final manuscript.

\section{Authors' information}

Not further applicable.

Competing interests

The authors declare that they have no competing interests.

Consent for publication

The samples for this retrospective study were preexisting archived paraffin blocks and no consent was required for publication of the deidentified results.

Ethics approval and consent to participate

The study was reviewed and approved by the local ethics committee at Wroclaw Medical University, Wroclaw, Poland. The pre-existing specimens did not require consent to participate.

\section{Author details}

${ }^{1}$ Department of Pathology, Wroclaw Medical University, Wroclaw, Poland. ${ }^{2}$ Department of General and Oncological Surgery, Wroclaw Medical University, Wroclaw, Poland. ${ }^{3}$ Division of Biostatistics and Epidemilogy, Cincinnati Children's Hospital Medical Center, Cincinnati, OH, USA. ${ }^{4}$ University of Cincinnati Academic Health Center, College of Pharmacy, 3225 Eden Avenue, Cincinnati, OH 45267-0004, USA. 
Received: 15 December 2015 Accepted: 4 July 2016

Published online: 11 July 2016

\section{References}

1. Pang H, Cai L, Yang Y, Chen X, Sui G, Zhao C. Knockdown of osteopontin chemosensitizes MDA-MB-231 cells to cyclophosphamide by enhancing apoptosis through activating p38 MAPK pathway. Cancer Biother Radiopharm. 2011;26:165-73.

2. Yang L, Wei L, Zhao W, Wang X, Zheng G, Zheng M, Song X, Zuo W. Downregulation of osteopontin expression by RNA interference affects cell proliferation and chemotherapy sensitivity of breast cancer MDA-MB-231 cells. Mol Med Rep. 2012;5:373-6.

3. Graessmann M, Berg B, Fuchs B, Klein A, Graessmann A. Chemotherapy resistance of mouse WAP-SVT/t breast cancer cells is mediated by osteopontin, inhibiting apoptosis downstream of caspase-3. Oncogene. 2007;26:2840-50.

4. Das S, Samant RS, Shevde LA. Nonclassical activation of Hedgehog signaling enhances multidrug resistance and makes cancer cells refractory to Smoothened-targeting Hedgehog inhibition. J Biol Chem. 2013;288:11824-33.

5. Martinetti A, Bajetta E, Ferrari L, Zilembo N, Seregni E, Del Vecchio M, Longarini R, La Torre I, Toffolatti L, Paleari D, Bombardieri E. Osteoprotegerin and osteopontin serum values in postmenopausal advanced breast cancer patients treated with anastrozole. Endocr Relat Cancer. 2004;11:771-9.

6. Yang L, Zhao W, Zuo WS, Wei L, Song XR, Wang XW, Zheng G, Zheng MZ. Silencing of osteopontin promotes the radiosensitivity of breast cancer cells by reducing the expression of hypoxia inducible factor 1 and vascular endothelial growth factor. Chin Med J (Engl). 2012;125:293-9.

7. Hahnel A, Wichmann H, Kappler M, Kotzsch M, Vordermark D, Taubert H, Bache M. Effects of osteopontin inhibition on radiosensitivity of MDA-MB231 breast cancer cells. Radiat Oncol. 2010;5:82.

8. Zduniak K, Ziolkowski P, Ahlin C, Agrawal A, Agrawal S, Blomqvist C, Fjällskog ML, Weber GF. Nuclear Osteopontin-C is a marker for breast cancer prognosis. Br J Cancer. 2015;112:729-38.

9. Dabbs DJ. Diagnostic Immunohistochemistry: Theranostic and Genomic Applications. Philadelphia (Saunders Elsevier). 3rd ed. 2010. p. 801-2

10. Patani N, Jiang W, Mokbel K. Osteopontin C mRNA expression is associated with a poor clinical outcome in human breast cancer. Int J Cancer. 2008; 122:2646.

11. Patani N, Jouhra F, Jiang W, Mokbel K. Osteopontin expression profiles predict pathological and clinical outcome in breast cancer. Anticancer Res. 2008;28:4105-10.

12. Sargent DJ, Conley BA, Allegra C, Collette L. Clinical trial designs for predictive marker validation in cancer treatment trials. J Clin Oncol. 2005;23:2020-7.

13. Mirza M, Shaughnessy E, Hurley JK, Vanpatten KA, Pestano GA, He B, Weber GF. Osteopontin-c is a selective marker for breast cancer. Int J Cancer. 2008;122: 889-97.

14. El-Tanani M, Fernig DG, Barraclough R, Green C, Rudland P. Differential modulation of transcriptional activity of estrogen receptors by direct protein-protein interactions with the $T$ cell factor family of transcription factors. J Biol Chem. 2001;276:41675-82.

15. Zirngibl RA, Chan JS, Aubin JE. Estrogen receptor-related receptor a (ERRa) regulates osteopontin expression through a non-canonical ERRa response element in a cell context-dependent manner. J Mol Endocrinol. 2008;40:61-73.

16. Welsh AW, Moeder CB, Kumar S, Gershkovich P, Alarid ET, Harigopal M, Haffty BG, Rimm DL. Standardization of estrogen receptor measurement in breast cancer suggests false-negative results are a function of threshold intensity rather than percentage of positive cells. J Clin Oncol. 2011;29:2978-84.

17. Thoms JW, Dal Pra A, Anborgh PH, Christensen E, Fleshner N, Menard C, Chadwick K, Milosevic M, Catton C, Pintilie M, Chambers AF, Bristow RG. Plasma osteopontin as a biomarker of prostate cancer aggression: relationship to risk category and treatment response. Br J Cancer. 2012; 107:840-6.

18. Mack PC, Redman MW, Chansky K, Williamson SK, Farneth NC, Lara Jr PN, Franklin WA, Le QT, Crowley JJ, Gandara DR. SWOG. Lower osteopontin plasma levels are associated with superior outcomes in advanced nonsmall-cell lung cancer patients receiving platinum-based chemotherapy: SWOG Study S0003. J Clin Oncol. 2008;26:4771-6.

19. Pectasides D, Papaxoinis G, Kalogeras KT, Eleftheraki AG, Xanthakis I, Makatsoris T, Samantas E, Varthalitis I, Papakostas P, Nikitas N, Papandreou CN, Pentheroudakis G, Timotheadou E, Koutras A, Sgouros J, Bafaloukos D, Klouvas G, Economopoulos T, Syrigos KN, Fountzilas G. XELIRI-bevacizumab versus FOLFIRI-bevacizumab as first-line treatment in patients with metastatic colorectal cancer: a Hellenic Cooperative Oncology Group phase III trial with collateral biomarker analysis. BMC Cancer. 2012;12:271.

20. Ramchandani D, Weber GF. Interactions between osteopontin and vascular endothelial growth factor: Implications for cancer. Biochim Biophys Acta. 2015;1855:202-22.

21. Hsieh IS, Huang WH, Liou HC, Chuang WJ, Yang RS, Fu WM. Upregulation of drug transporter expression by osteopontin in prostate cancer cells. Mol Pharmacol. 2013;83:968-77.

\section{Submit your next manuscript to BioMed Central and we will help you at every step:}

- We accept pre-submission inquiries

- Our selector tool helps you to find the most relevant journal

- We provide round the clock customer support

- Convenient online submission

- Thorough peer review

- Inclusion in PubMed and all major indexing services

- Maximum visibility for your research

Submit your manuscript at www.biomedcentral.com/submit
C Biomed Central 